\title{
Acute gastritis associated with invading Helicobacter heilmannii organisms from a previously homeless cat
}

Hiroshi Ohtani, Hiroko Hayashi, Yoshikazu Higami, Kazuhiko Takuma, Kenjiro Minami, Yasuhiro Minami, Isao Shimokawa

Department of Pathology, Kitakyushu City Yahata Hospital (H Ohtani M.D.), Department of Investigative Pathology, Nagasaki University Graduate School of Biomedical Science (H Hayashi M.D., Y Higami M.D., Prof I Shimokawa M.D.), Takuma Clinic (K Takuma M.D.), and Minami Dog and Cat Hospital (K Minami veterinarian, Y Minami veterinarian)

Correspondence to:

Hiroshi Ohtani, M.D.

4-18-1 Nishihonmachi, Yahatahigashiku, Kitakyushu-city, Fukuoka 805-8534, Japan

Phone: +81-93-662-6565

Fax: +81-93-662-1796

e-mail: ohtani@yahatahp.jp 
To the Editor:

$H$. heilmannii are unculturable, and gram-negative rod, apparently larger than $H$. pylori, and are not usually (frequency 0.1-6.2\%) observed in routine gastric biopsy. ${ }^{1,2}$ Most infected stomachs show only mild gastritis but rarely is it associated with acute gastritis, peptic ulcer, gastric carcinoma or mucosa-associated lymphoid tissue lymphoma., ${ }^{2,3}$ Although $H$. heilmannii exclusively floats in the mucous layer above the surface and foveolar epithelial cells, Heilmann and Borchard using transmission electron microscopy showed that the organisms could attach to and penetrate into gastric parietal cells. ${ }^{1}$ Using giemsa stain and immunostaining, we first report that $H$. heilmannii can penetrate to gastric epithelial cells and further invade into the stroma.

A previously healthy 58-year-old man presented with epigastric pain. Gastric endoscopy showed severe hemorrhage and erosion in the body and the antrum of his stomach. A biopsy taken from body mucosa showed erosion, hemorrhage, marked infiltration of polymorphonuclear leukocytes (neurtophils), and glandular destruction (Fig. 1a). Giemsa stain revealed clusters of corkscrew-shaped bacilli in the surface mucous layer and some foveolae. The organisms were positive for polyclonal anti-Helicobacter pylori antibody (DakoCytomation, Kyoto, Japan), consistent with Helicobacter (H.) heilmannii. Spiral bacilli were also present within foveolar epithelial cells and in the stroma (Fig. 1a inset). He underwent antimicrobial treatment by oral administration of lansoplazole, amoxillin and clarithromycin; simulating the standard treatment for $H$. pylori gastritis. Re-endoscopy two weeks after the initial gastroscopy showed an improvement of his acute gastritis and the disappearance of the organisms; however, two healing 
linear ulcers were retained in his antral mucosa. Gastroscopy and biopsy six months after the initial endoscopy showed no evidence of abnormal mucosal lesions or H. heilmannii infection.

For over three years he had kept in his house three healthy pet dogs with good pedigrees obtained from breeders. Several months before the initial gastroscopy, he picked up a healthy but then homeless cat and kept it in his restaurant that was separated from his house. The dogs and the cat were anesthetized and underwent gastroscopy with the consent of the patient. Biopsy from all the animals revealed minimal to moderate gastritis with the specimens from the cat showing spiral bacilli presenting in the mucous layer, gastric foveolae, and cytoplasm of parietal cells with intracytoplasmic vacuolation around the organisms (Fig. 1b). They also cross immunostained with anti-H. pylori antibody.

The frequency of $H$. heilmannii infection in dogs is 67 to $100 \%$ and in cats it is 42 to $100 \%,{ }^{4}$ while its infection in humans has been postulated to be an example of zoonosis. ${ }^{5}$ The patient's dogs that lived in his house were not infected with the organism because they had been kept apart from the infected cat kept that lived in his restaurant. This suggests that $H$. heilmannii infection between animals is connected with poor sanitary conditions in which infected animals live together. Owners of pet animals that have grown up in a clean environment are not always oversensitive to $H$. heilmannii infection. This case of acute $H$. heilmannii gastritis was almost certainly caused by organisms derived from patient's cat; if so, the incubation period of the disease may be presumed to have been within several months. The mechanism by which indolent organisms in a cat could cause acute gastritis in a human stomach is unknown; however, a possible explanation may 
include that a potentially aggressive strain of $H$. heilmannii penetrated into the epithelial cells and the stroma resulting in neutrophilic infiltration and subsequent glandular destruction. We concluded that invading $H$. heilmannii organisms could cause acute gastritis in humans when animals with the organisms are kept as a pet.

\section{Acknowledgments}

The authors want to thank Mr Junji Hisano and Ms Yuko Moriyama for preparation of the pathological specimens.

\section{References}

1. Heilmann KL, Borchard F. Gastritis due to spiral shaped bacteria other than Helicobacter pylori: clinical, histological, and ultrastructural findings. Gut 1991; 32:137-40.

2. Singhal AV, Sepulveda AR. Helicobacter heilmannii gastritis A case study with review of literature. Am J Surg Pathol 2005; 29:1537-1539.

3. Lavelle JP, Landas S, Mitros FA, et al. Acute gastritis associated with spiral organisms from cats. Dig Dis Sci 1994; 39:744-750.

4. Eaton KA, Dewhirst FE, Paster BJ, et al. Prevalence and varieties of helicobacter species in dogs from random sources and pet dogs: animal and public health implications. J Clin Microbiol 1996; 34:3165-3170.

5. Thomson MA, Storey P, Greer R, et al. Canine-human transmission of Gastrospirillum hominis. Lancet 1994; 343:1605-1606. 


\section{Figure legend}

FIG. 1 (a) Gastric histology of the patient (magnification $\times 100$ ); the inset highlights spiral $H$. heilmannii organisms (arrows) in the destructed foveolae and in the stroma ( $\times 1000$, anti-H. pylori immunostaining). (b) Histology of the previously homeless cat showing colonized organisms (arrows) in the cytoplasm of parietal cells $(\times 1000$, Giemsa).

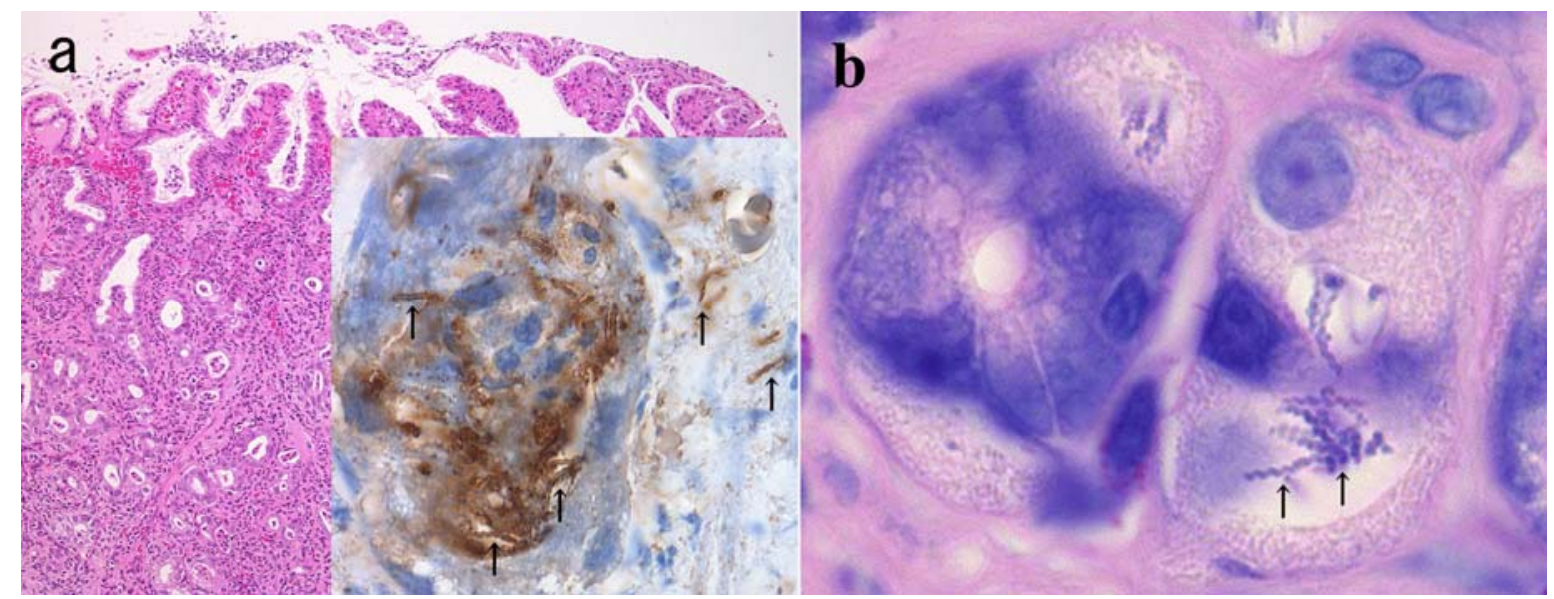

